$\mathbb{T}$ periodica polytechnica

Civil Engineering

$51 / 2(2007) 51+55$

doi: 10.3311/pp.ci.2007-2.08

web: http://www.pp.bme.hu/ci

(c) Periodica Polytechnica 2007

RESEARCH ARTICLE

\section{Elastic-plastic optimum design of} frames taking into account semi-rigid connections

\author{
Péter Nédli / Alain Lachal
}

Received 2007-11-14

\begin{abstract}
Classical rigid plastic optimum design cannot take into account displacement constraints or bounds on residual plastic strains which goal however maybe important when designing a structure. Using holonomic elastic-plastic constitutive law, the elastic-plastic optimum design problem can be formulated on the basis of the elastic-plastic analysis problem like the rigid plastic optimum design problem is based on rigid plastic limit analysis. In this way, the elastic properties can be included in the optimum design problem and also semi-rigid connections can be taken into account. This paper deals with several elasticplastic optimum design problems, namely, geometrically linear and nonlinear holonomic elastic-plastic optimum design and geometrically linear shakedown optimum design. Each of these problems leads to nonlinear programming. The different design problems are illustrated on a sample frame by using the MINOS mathematical programming package for the solution.
\end{abstract}

\section{Keywords}

holonomic elastic-plastic optimum design $\cdot$ geometrical nonlinearity · semi-rigid connection $\cdot$ shakedown optimum design . nonlinear programming

\section{Acknowledgement}

The financial support of grant TÉT F-44/05 is gratefully acknowledged.

\section{Péter Nédli}

Department of Structural Mechanics, BME, H-1111 Budapest, Múegyetem rkp. 3 , Hungary

Alain Lachal

LGCGM, INSA de Rennes, 20 avenue des Buttes de Coësmes, 35043 Rennes cedex, France

\section{Governing relations for the holonomic elastic-plastic analysis problem}

A discrete approach is accepted for the description of the structural model. The material is assumed to be linear elastic perfectly plastic. Linearized yield condition depending only on the bending moment is used with associated flow rules. The governing vector variables are the nodal loads $(\mathbf{q})$, the generalized initial strains ( $\mathbf{t}$ ), the nodal displacements (u), the internal forces (s), the plastic loadbearing capacities i.e. the plastic yield moments $(\mathbf{k})$, the plastic potential $(\boldsymbol{\varphi})$ and the plastic multiplier $(\lambda)$. Semi-rigid connections are taken into account as linear elastic perfectly plastic rotational springs [1], [2]. Before detailing the optimum design problems, the corresponding analysis problems are summarized.

\subsection{First order holonomic elastic-plastic analysis}

In order to be able to transform the analysis problem into an optimum design problem, the holonomic elastic-plastic constitutive law is used (i.e. local unloading is discarded during the load history) which is usually acceptable in the case of monotonic one parameter loading. The governing relations for first order holonomic elastic-plastic limit analysis are the following:

$$
\begin{gathered}
{\left[\begin{array}{ccccc}
\mathbf{0} & \mathbf{G} & \mathbf{0} & \mathbf{0} & \mathbf{q}_{\mathrm{b}} \\
\mathbf{G}^{\mathrm{T}} & \mathbf{F} & \mathbf{N}^{\mathrm{T}} & \mathbf{0} & \mathbf{t}_{\mathrm{b}} \\
\mathbf{0} & \mathbf{N} & \mathbf{0} & -\mathbf{E} & \mathbf{0}
\end{array}\right]\left[\begin{array}{c}
\mathbf{u} \\
\mathbf{s} \\
\lambda \\
\boldsymbol{\varphi} \\
m
\end{array}\right]+\left[\begin{array}{c}
\mathbf{0} \\
\mathbf{0} \\
\mathbf{k}
\end{array}\right]=\mathbf{0}} \\
\lambda \geq \mathbf{0}, \quad \boldsymbol{\varphi} \leq \mathbf{0}, \quad \boldsymbol{\varphi}^{\mathrm{T}} \lambda=0, \quad m=\max !
\end{gathered}
$$

Here $m$ is the load parameter, $\mathbf{E}$ is the unit matrix, index ' $\mathrm{b}$ ' refers to the base load i.e. $\mathbf{q}=m \cdot \mathbf{q}_{\mathbf{b}}, \mathbf{G}$ is the equilibrium matrix, $\mathbf{F}$ is the flexibility matrix and $\mathbf{N}$ is the yield matrix. The first equation is the equilibrium equation, the second is the compatibility equation (joint geometrical and physical equation) and the third one is the yield condition. The fourth line expresses the sign constraints on the plastic variables, the normality rule between them and the objective function is the load parameter. 


\subsection{Geometrically nonlinear holonomic elastic-plastic} analysis

Due to possible instability failure in case of sway frames, the real limit load is less than the one given by the first order elastic-plastic analysis. To account for this fact, geometrically nonlinear elastic-plastic analysis can be done. There are several methods to take into account geometrical nonlinearity. In this paper we use the method described in [3] for elastic bar structures based on the Lagrangian approach. The main idea of the method is that if one knows the displacements and the internal forces at the beginning of an elastic beam which has only nodal loads, then a first order initial value differential equation system can be written to determine its shape. Plastic hinges and semirigid joints can be taken into account in the initial conditions. As the system of differential equations can be solved only numerically, the governing relations cannot be written in a matrix form but only in the form of nonlinear vector-vector functions.

$$
\begin{gathered}
\mathbf{e}(\mathbf{v}, \mathbf{s}, m)=\mathbf{0} \\
\mathbf{c}(\mathbf{v}, \mathbf{s}, \lambda, m)=\mathbf{0} \\
\mathbf{y}(\mathbf{v}, \mathbf{s}, \lambda, \boldsymbol{\varphi})=\mathbf{k} \\
\lambda \geq \mathbf{0} \quad \boldsymbol{\varphi} \leq \mathbf{0}, \quad \boldsymbol{\varphi}^{\mathrm{T}} \boldsymbol{\lambda}=0, \quad m=\max !
\end{gathered}
$$

Here e, $\mathbf{c}, \mathbf{y}$ refer respectively to the equilibrium, compatibility and yield equations. At a given value of the state variables, these equations can be evaluated numerically. This is sufficient for the MINOS nonlinear mathematical programming solver as it can compute the Jacobian of the nonlinear system by numerical derivation. This system of equations can be built by the usual way i.e. by assembly of the equations of the elements. Fig. 1 shows the variables for one element. Shear and axial deformation usually contribute little to the displacements so

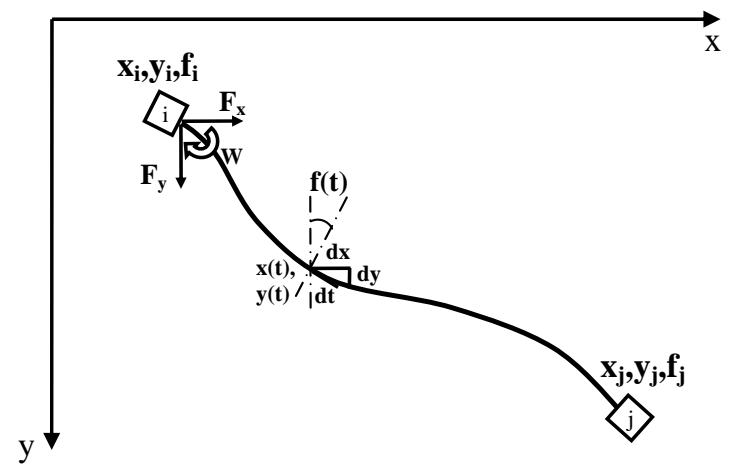

Fig. 1. Variables for one elastic element

they can be neglected and then the initial value differential equation system which describes the displaced configuration of an initially straight beam between the nodes $\mathrm{i}, \mathrm{j}$ having $\alpha$ angle to the $x$ axis and length $\ell$ is the following:

$$
\begin{aligned}
& \frac{\mathrm{d} x(t)}{\mathrm{d} t}=\cos (f(t)), \quad \frac{\mathrm{d} y(t)}{\mathrm{d} t}=\sin (f(t)), \\
& \frac{\mathrm{d} f(t)}{\mathrm{d} t}=\frac{1}{E I}\left(W+F_{x}\left(y(t)-y_{\mathrm{i}}\right)-F_{y}\left(x(t)-x_{\mathrm{i}}\right)\right. \\
& \text { with the initial conditions: } x(0)=x_{\mathrm{i}}, \quad y(0)=y_{\mathrm{i}}, \\
& f(0)=f_{\mathrm{i}}+\alpha+\lambda_{\mathrm{i}}^{+}-\lambda_{\mathrm{i}}^{-}+c_{\mathrm{i}} W
\end{aligned}
$$

Here, $F_{x}, F_{y}, W$ represent the internal forces of the element, $x_{\mathrm{i}}$, $y_{\mathrm{i}}, f_{\mathrm{i}}, x_{\mathrm{j}}, y_{\mathrm{j}}, f_{\mathrm{j}}$ are the coordinates of the nodes of the element ( $f$ refers to the rotation), $t$ is the arclength variable going from 0 to $\ell, c_{\mathrm{i}}$ is the spring coefficient of the semi-rigid connection and $\lambda_{i}^{+}, \lambda_{i}^{-}$are the plastic multipliers. This differential equation system can be solved numerically and the values of $x(\ell), y(\ell)$, $f(\ell)$ can be obtained so the contribution of the element to the equilibrium, compatibility and yield condition can be evaluated. In fact, there are 12 equations for the element: 6 equilibrium, 3 compatibility and 4 yield equations. The equilibrium equations are the only contributions which should be assembled for all nodes but the others are element equations and as such complete. These equations are the following:

$\mathrm{e}_{1}: F_{x}, \quad \mathrm{e}_{2}: F_{y}, \quad \mathrm{e}_{3}: W, \quad \mathrm{e}_{4}: F_{x}, \mathrm{e}_{5}: F y$

$\mathrm{e}_{6}: F_{x}\left(y_{\mathrm{j}}-y_{\mathrm{i}}\right)+F_{y}\left(x_{\mathrm{j}}-x_{\mathrm{i}}\right)-W$,

$\mathrm{c}_{1}: x(\ell)-x_{2}=0, \quad \mathrm{c}_{2}: y(\ell)-y_{2}=0$,

$c_{3}: f(\ell)-\alpha+\lambda_{\mathrm{j}}^{+}-\lambda_{\mathrm{j}}^{-}+c_{\mathrm{j}}\left(F_{x}\left(y_{\mathrm{j}}-y_{\mathrm{i}}\right)+F_{y}\left(x_{\mathrm{j}}-x_{\mathrm{i}}\right)-W\right)-f_{\mathrm{j}}=0$

$\mathrm{y}_{1}: W-\varphi_{\mathrm{i}}^{+}-M_{y \mathrm{i}}^{+}=0, \quad \mathrm{y}_{2}: W-\varphi_{\mathrm{i}}^{-}-M_{y \mathrm{i}}^{-}=0$,

$\mathrm{y}_{3}: W_{\mathrm{j}}-\varphi_{\mathrm{j}}^{+}-M_{y \mathrm{j}}^{+}=0, \quad \mathrm{y}_{4}: W_{\mathrm{j}}-\varphi_{\mathrm{j}}^{-}-M_{y \mathrm{j}}^{\mathrm{i}-}=0$

Here $W_{\mathrm{j}}$ is the bending moment at node $\mathrm{j}$ which is: $W_{\mathrm{j}}=$ $F_{x}\left(y_{\mathrm{j}}-y_{\mathrm{i}}\right)-F_{y}\left(x_{\mathrm{j}}-x_{i}\right)-W$.

\section{Geometrically linear holonomic elastic-plastic opti- mum design}

In the same way as plastic optimum (minimum weight) design can be derived from plastic limit analysis, the holonomic elastic-plastic analysis can be transformed into an optimum design problem. The goal is to find the structure which weight is minimal and which is in plastic limit state under the given load. As this approach also computes the displacements, bounds can be given on the maximum displacements. The objective function is the so called relative weight of the structure which takes as specific weight the necessary design moments of the frame. Using the notations of Eurocode [4], [5], it can be expressed as $w=\sum L_{k} M_{\mathrm{pl}, \mathrm{Rd}, k}$, where $M_{\mathrm{pl}, \mathrm{Rd}, k}$ is the design plastic resistance moment $\left(M_{\mathrm{pl}, \mathrm{Rd}}\right)$ of the $k^{\text {th }}$ cross section type and $L_{k}$ is the total length of the members of the $k^{\text {th }}$ cross section type. As the elastic properties are also used in this formulation, some simplifying assumptions are made. It is assumed that the depth $(h)$ of the different cross-section types is fixed. Sections are assumed to be $I$ sections and their web is neglected. So the the design plastic resistance moment of a section is $M_{\mathrm{pl}, \mathrm{Rd}, k}=A_{f} \sigma_{y} h$, its second moment of area is $I=A_{f} h^{2} / 2$, so $A_{f}=M_{\mathrm{pl}, \mathrm{Rd}, k} /\left(\sigma_{y} h\right), I=\left(M_{\mathrm{pl}, \mathrm{Rd}, k} h\right) /\left(2 \sigma_{y}\right)$ and by this way the only design variables are the plastic limit moments $\left(M_{\mathrm{pl}, \mathrm{Rd}, k}\right)$ of the different section types. The vector of their values is denoted by $\boldsymbol{\kappa}$. As the number of the design variables is usually much less than the number linearized yield conditions, the vector $\mathbf{k}$ of the yield capacities can be written in the form $\mathbf{k}=\mathbf{M} \boldsymbol{\kappa}$, where $\mathbf{M}$ is a matrix which maps $\boldsymbol{\kappa}$ into $\mathbf{k}$. It is also assumed that the properties of the semi rigid connections (rotational stiffness, resistance moment) are fixed and thus the optimization is done only for the design limit moments of 
elements. The design constrains applied are: ratios among the design variables $(\mathbf{R} \boldsymbol{\kappa} \leq \mathbf{0})$ to avoid that columns on the lower levels be weaker than on the upper levels, bounds on the displacements and on the plastic strains as well as bound on the plastic dissipation $\left(W_{\mathrm{p}}\right)$. So the optimum design problem is formulated as follows:
equilibrium:
$\mathbf{G s}+\mathbf{q}=\mathbf{0}$
compatibility:
$\mathbf{G s} \mathbf{s}^{\mathrm{T}}+\mathbf{F}(\boldsymbol{\kappa}) \mathbf{s}+\mathbf{p}+\mathbf{t}=\mathbf{0}$
yield condition:
Ns $-\varphi-\mathbf{M} \kappa=\mathbf{0}, \varphi \leq \mathbf{0}$
flow rules:
$\mathbf{p}=\mathbf{N}^{\mathrm{T}} \lambda, \lambda \geq \mathbf{0}, \boldsymbol{\varphi}^{\mathrm{T}} \lambda=0$
design constrains:
$\boldsymbol{\kappa} \geq \mathbf{0}, \mathbf{R} \boldsymbol{\kappa} \leq \mathbf{0}, \mathbf{u}_{\mathrm{b}}^{-} \leq \mathbf{u} \leq \mathbf{u}_{\mathrm{b}}^{+}$,
$\mathbf{p}_{\mathrm{b}}^{-} \leq \mathbf{N}^{\mathrm{T}} \boldsymbol{\lambda} \leq \mathbf{p}_{\mathrm{b}}^{+}, \lambda^{\mathrm{T}} \mathbf{M} \boldsymbol{\kappa} \leq W_{\mathrm{pb}}$
objective function: $\mathbf{h}^{\mathrm{T}} \boldsymbol{\kappa}=\min$ !

As the flexibility matrix depends on the design variables, so this is a general nonlinear mathematical programming problem. In order that the first order design be realistic, it would be necessary to include stability requierements described in the codes [4], [5]. Because of this, in this paper we concentrate rather on the geometrically nonlinear design in which the stability requirements are included indirectly (second order theory).

\section{Geometrically nonlinear holonomic elastic-plastic optimum design}

Taking into account geometrical nonlinearity according to section 1.2, the design problem can be formulated as follows:

$$
\begin{array}{ll}
\text { equilibrium: } & \mathbf{e}(\mathbf{v}, \mathbf{s}, \mathbf{q})=\mathbf{0} \\
\text { compatibility: } & \mathbf{c}(\mathbf{v}, \mathbf{s}, \boldsymbol{\lambda}, \mathbf{t}, \boldsymbol{\kappa})=\mathbf{0} \\
\text { yield condition: } & \mathbf{y}(\mathbf{v}, \mathbf{s}, \lambda, \boldsymbol{\varphi}, \boldsymbol{\kappa})=\mathbf{0}, \boldsymbol{\varphi} \leq \mathbf{0} \\
\text { flow rules: } & \mathbf{p}=\mathbf{N}^{\mathrm{T}} \lambda, \boldsymbol{\lambda} \geq \mathbf{0}, \boldsymbol{\varphi}^{\mathrm{T}} \lambda=0 \\
\text { design constraints: } & \boldsymbol{\kappa} \geq \mathbf{0}, \mathbf{R} \boldsymbol{\kappa} \leq \mathbf{0}, \mathbf{u}_{\mathrm{b}}^{-} \leq \mathbf{u} \leq \mathbf{u}_{\mathrm{b}}^{+}, \\
& \mathbf{p}_{\mathrm{b}}^{-} \leq \mathbf{N}^{\mathrm{T}} \lambda \leq \mathbf{p}_{\mathrm{b}}^{+}, \lambda^{\mathrm{T}} \mathbf{M} \boldsymbol{\kappa} \leq W_{\mathrm{pb}}, \boldsymbol{\kappa} \geq \mathbf{0}
\end{array}
$$

objective function: $\mathbf{h}^{\mathrm{T}} \boldsymbol{\kappa}=\min$ !

Similarly to the geometrically nonlinear elastic-plastic analysis, the equilibrium, compatibility and yield equations can be evaluated only numerically through the solution of differential equations.

\section{Geometrically linear shakedown optimum design}

In the case of shakedown, the departure point is the determination of the fictitious generalized elastic stresses due to the load history [6]. The usual load system used in shakedown is composed of ' $n$ ' independent load cases. Within one load-case, the load values can vary proportionally from zero to their maximal value. For the load history, it is assumed that all possible combinations of the independent load cases can happen with any intermediate value between zero and their maximum. In fact, what one needs from the load history is $\varphi_{\mathrm{e}}^{\max }=\mathbf{N s}_{\mathrm{e}}(t)$, i.e. the maximum values of the yield function during the load history. Here, $t$ represents the time symbolically. If one uses the load system described above, then the $i^{\text {th }}$ element of $\varphi_{\mathrm{e}}^{\max }$ is the following:

$$
\varphi_{\mathrm{e}, i}^{\max }=\sum_{j=1}^{n}\left\{\max \left(\mathbf{n}_{i}^{\mathrm{T}} \mathbf{s}_{\mathrm{e}, j}\right) \mid \mathbf{n}_{i}^{\mathrm{T}} \mathbf{s}_{\mathrm{e}, j}>0\right\}
$$

Here $\mathbf{s}_{\mathrm{e}, j}$ means the generalized stresses from the $j^{\text {th }}$ load-case, $\mathbf{n}_{i}^{\mathrm{T}}$ is the $i^{\text {th }}$ row of the yield matrix $(\mathbf{N})$. In fact, one has to make a conditional sum taking into account only those values which contribute to the activation of the $i^{\text {th }}$ yield condition.

Based on the statical shakedown theorem, the shakedown optimum design problem is as follows:

$$
\begin{array}{ll}
\text { equilibrium: } & \mathbf{G s}=\mathbf{0} \\
\text { compatibility: } & \mathbf{G}^{\mathrm{T}} \mathbf{u}+\mathbf{F}(\boldsymbol{\kappa}) \mathbf{s}+\mathbf{p}+\mathbf{t}=\mathbf{0} \\
\text { yield condition: } & \mathbf{N s}+\boldsymbol{\varphi}_{\mathrm{e}}^{\max }(\boldsymbol{\kappa})-\boldsymbol{\varphi}-\mathbf{M} \boldsymbol{\kappa}=\mathbf{0}, \boldsymbol{\varphi} \leq \mathbf{0} \\
\text { flow rules: } & \mathbf{p}=\mathbf{N}^{\mathrm{T}} \lambda, \lambda \geq \mathbf{0}, \boldsymbol{\varphi}^{\mathrm{T}} \boldsymbol{\lambda}=0 \\
\text { design constrains: } & \boldsymbol{\kappa} \geq \mathbf{0}, \mathbf{R} \boldsymbol{\kappa} \leq \mathbf{0}, \mathbf{u}_{\mathrm{b}}^{-} \leq \mathbf{u} \leq \mathbf{u}_{\mathrm{b}}^{+}, \\
& \mathbf{p}_{\mathrm{b}}^{-} \leq \mathbf{N}^{\mathrm{T}} \boldsymbol{\lambda} \leq \mathbf{p}_{\mathrm{b}}^{+}, \lambda^{\mathrm{T}} \mathbf{M} \boldsymbol{\kappa} \leq W_{\mathrm{pb}}
\end{array}
$$

objective function: $\mathbf{h}^{\mathrm{T}} \boldsymbol{\kappa}=\min$ !

By giving a bound on the plastic dissipation work, one can control the design going from fully elastic behavior $\left(W_{\mathrm{pb}}=0\right)$ to the adaptation limit (no constraint $W_{\mathrm{pb}}$ but it should be finite).

\section{Numerical solution and example}

To solve the optimization problems, the MINOS mathematical programming system was used. A familiar industrial 4storey, single-bay steel frame was considered, previously examined in references [7], [8] for elastic-plastic analysis. Its geometry, intensities of the loading including roof, floors, wind pressure are given in Fig. 1. This load is associated to the value 1.00 of the load parameter $(m)$.

Tab. 1. Cross-section characteristics

\begin{tabular}{lccc}
\hline $\begin{array}{l}\text { Section } \\
\text { Ref. }\end{array}$ & $\begin{array}{c}\text { Cross-section } \\
\text { area }\left(\mathrm{cm}^{2}\right)\end{array}$ & $\begin{array}{l}\text { Second moment } \\
\text { of area }\left(\mathrm{cm}^{4}\right)\end{array}$ & $\begin{array}{l}\text { Plastic resistance } \\
\text { moment }(\mathrm{kN} \cdot \mathrm{m})\end{array}$ \\
\hline A1 & 34.19 & 2315 & 61.98 \\
A2 & 47.62 & 5092 & 108.71 \\
A3 & 38.00 & 1445 & 52.15 \\
A4 & 47.55 & 1819 & 65.85 \\
A5 & 53.42 & 3608 & 99.95 \\
A6 & 66.58 & 4789 & 127.58 \\
\hline
\end{tabular}

Yield strength and modulus of elasticity of the steel frame are $225 \mathrm{MPa}$ and $210000 \mathrm{MPa}$, respectively. Table 1 gives: cross-section area, second moment of area and plastic moment resistance for each frame member. In $1^{\text {st }}$ order and $2^{\text {nd }}$ order analysis, hypothesis of a radial loading was made, meanwhile for shakedown optimum design, all the eight concentrated forces were allowed to vary independently from each other.

A first comparison of the results deals with the limit load multiplier obtained for the three types of analysis considered in this study:

- a normal elastic analysis corresponding to $m=1.00$;

- a first order holonomic elastic-plastic analysis [8] which gives $m_{\max }=2.24$; the same as the one obtained from a classical rigid-plastic limit analysis, but in addition, in the case of holonomic elastic-plastic approach, values of nodal displacements are known. The maximum drift (at the top of the frame) is $u_{\text {max }}=0.8130 \mathrm{~m}$. 
- a second order nonlinear holonomic elastic-plastic analysis, where $m_{\max }$ is found equal to 2.044 and the maximum drift (at the top of the frame): $u_{\max }=0.3236 \mathrm{~m}$.

Besides the normal increasing of multiplier $m$ between value 1.00 of the elastic analysis which corresponds to a limit serviceability state and values 2.24 and 2.04 which correspond to an ultimate limit state, it is to underline that additional results in lateral displacement are obtained from the holonomic approach. Comparison between $1^{\text {st }}$ and $2^{\text {nd }}$ order analysis shows a difference between the maximum drift values. The drift obtained by second order analysis is smaller as the structure reaches the limit point of its loadbearing capacity well before the formation of a mechanism. However, the frame of Fig. 2 appears rather rigid and would not require a $2^{\text {nd }}$ order analysis (application of EN 1993-1-1 specifications [4] would lead to the same conclusion).

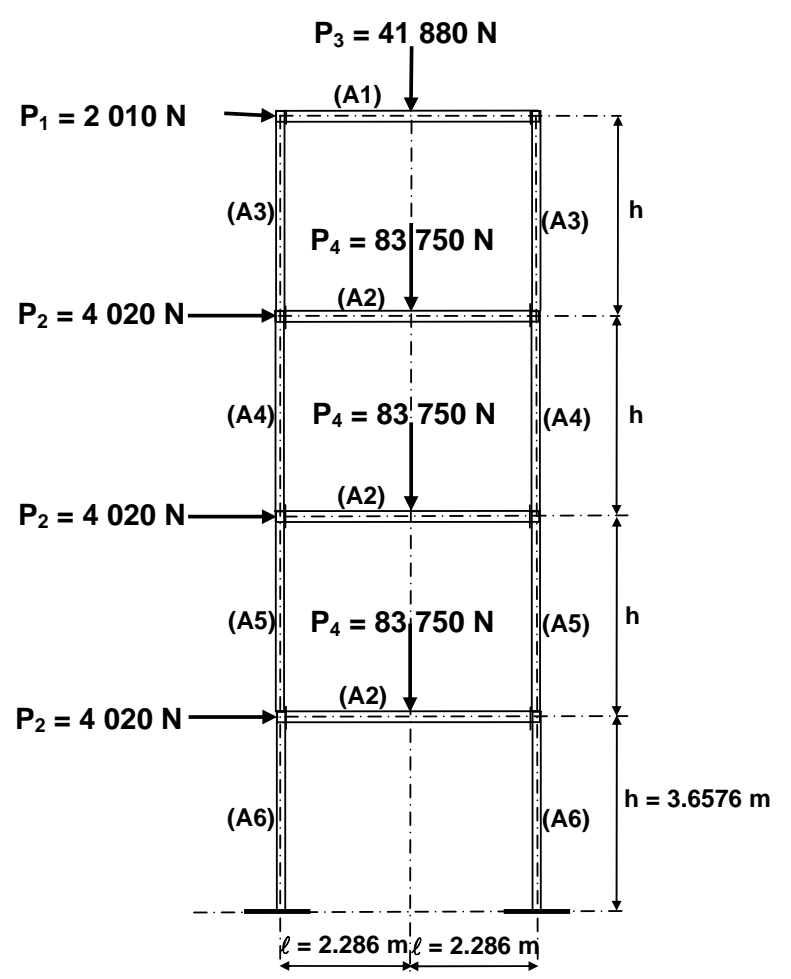

Fig. 2. Geometry and loads of the sample frame

A second comparison of the results deals with the influence of the joint behavior [9] on elastic-plastic limit values obtained from $1^{\text {st }}$ and $2^{\text {nd }}$ order holonomic analysis approaches. According to the joint classification given in Eurocode 3, three assumptions were made and presented in Table 2 . In the previous results presented here above, the assumption was made that the joints were rigid and full strength. In this part of the study, joints are always considered as full strength but three different values of rotational stiffness are studied. Considering classification boundaries given in 5.5.2.5. of EN 1993-1-8 (Fig. 3), three rotational stiffnesses have been selected: the rigid bound value for sway frames: $25 E I_{\mathrm{b}} / \mathrm{L}_{\mathrm{b}}$ the rigid bound value for fixed frames: $8 E I_{\mathrm{b}} / L_{\mathrm{b}}$ and an intermediate semi-rigid value of $16.5 E I_{\mathrm{b}} / L_{\mathrm{b}}$. The nominally pinned bound value $0.5 E I_{\mathrm{b}} / L_{\mathrm{b}}$ was left out of the study as the structure would become two weak against horizontal sway.

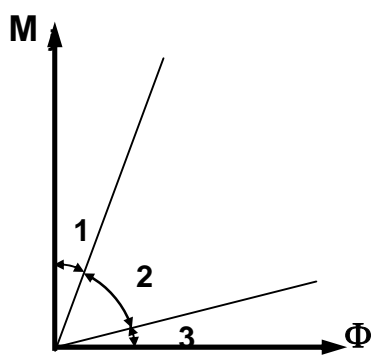

Fig. 3. Rotational stiffness classification (according to Eurocode 3)

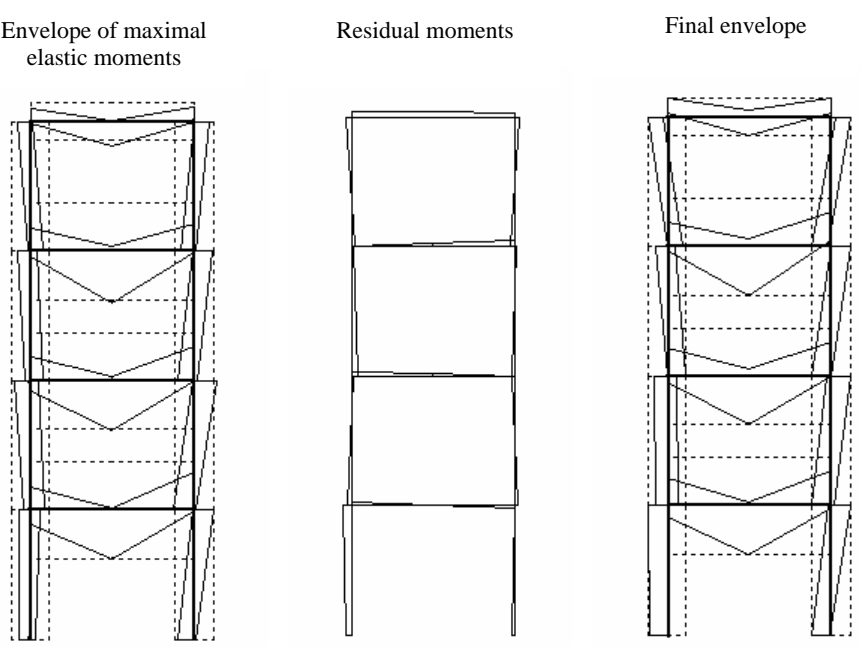

Fig. 4. Moment diagrams of shakedown for fixed connections

A third comparison of the results, presented in Table 3, deals with a comparative study of the results of the various optimum design problems including the original structure as well. The design load used was the geometrically nonlinear plastic limit load of the original structure. The design variables are the plastic yield moments of the different section types. Six different section types were considered accordingly to the original structure. These are the plastic yield moments of the upmost beam $\left(\mathrm{My}_{1}\right)$, the other beams $\left(\mathrm{My}_{2}\right)$, and columns story by story $\left(\mathrm{My}_{3}, \mathrm{My}_{4}\right.$, $\mathrm{My}_{5}, \mathrm{My}_{6}$ ). To compute the areas and the inertia moments of the sections from the plastic moments, the original section depths were used, namely $0.165,0.205,0.125,0.125,0.165,0.165$ all in meter. The yield stress used is $225 \mathrm{MPa}$. The semi-rigid connections were taken into account with a rotational rigidity of 25 , 16.5 , and $8 E I_{\mathrm{b}} / L_{\mathrm{b}} \mathrm{kNm} / \mathrm{m}$.

The nominal pinned connection would make the structure too weak against the horizontal sway, so this case was not studied. The meaning of the letters and numbers in the names given to the different designs is: $\mathrm{H}$ : holonomic, $\mathrm{SH}$ : shakedown, F: fixed, $\mathrm{S}$ : semi-rigid connection. Holonomic designs and checks were done by geometrically nonlinear analysis. All the designs were checked by holonomic and shakedown analysis. The shakedown drifts $\left(u_{\text {shrmax }}\right)$ given are the ones due to the residual stresses. The units are in $[\mathrm{kN}]$ and $[\mathrm{m}]$. It can be seen that the semi-rigid 
Tab. 2. Joint rotational stiffnesses and analysis results

\begin{tabular}{|c|c|c|c|c|c|c|c|c|}
\hline \multirow{3}{*}{ Joint behaviour } & \multicolumn{2}{|c|}{ Rotational stiffnesses (kNm/rad) } & \multicolumn{6}{|c|}{ Analysis results } \\
\hline & \multirow{2}{*}{ Two top angle joints } & \multirow{2}{*}{ Other $T$ joints } & \multicolumn{2}{|c|}{ First order elastic } & \multicolumn{2}{|c|}{ First order elasto-plastic } & \multicolumn{2}{|c|}{ Second order elasto-plastic } \\
\hline & & & $m$ & $u_{\max }(\mathrm{m})$ & $m$ & $u_{\max }(\mathrm{m})$ & $\mathrm{m}$ & $u_{\max }(\mathrm{m})$ \\
\hline Limit rigid (25) & 26096 & 56867 & 1.00 & 0.0245 & 2.2432 & 0.8130 & 2.0378 & 0.3198 \\
\hline Semi-rigid (16.5) & 17223 & 37532 & 1.00 & 0.0258 & 2.2432 & 0.8130 & 2.0378 & 0.3206 \\
\hline Limit(sway)semi-rigid (8) & 8351 & 18198 & 1.00 & 0.0296 & 2.2432 & 0.8130 & 2.0378 & 0.3230 \\
\hline
\end{tabular}

Tab. 3. Design results for different semi-rigid connections

\begin{tabular}{|c|c|c|c|c|c|c|c|c|c|}
\hline structure & original & $\mathrm{HF}$ & HS25 & HS16,5 & HSM8 & SHF & SHS25 & SHS16,5 & SHS8 \\
\hline $\mathrm{My}_{1}$ & 61.98 & 58.18 & 55.98 & 56.32 & 57.63 & 53.59 & 53.02 & 52.76 & 52.76 \\
\hline $\mathrm{My}_{2}$ & 108.71 & 118.99 & 118.73 & 118.60 & 118.17 & 137.53 & 136.53 & 135.85 & 135.85 \\
\hline $\mathrm{My}_{3}$ & 52.15 & 42.15 & 43.08 & 42.67 & 41.20 & 51.84 & 52.40 & 52.76 & 52.76 \\
\hline $\mathrm{My}_{4}$ & 65.86 & 54.70 & 55.50 & 55.85 & 57.12 & 51.84 & 52.40 & 52.76 & 52.76 \\
\hline $\mathrm{My}_{5}$ & 99.95 & 59.50 & 59.36 & 59.30 & 59.08 & 51.84 & 52.40 & 52.76 & 52.76 \\
\hline $\mathrm{My}_{6}$ & 127.58 & 59.50 & 59.36 & 59.30 & 59.08 & 51.84 & 52.40 & 52.76 & 52.76 \\
\hline weight & 4238.39 & 3476.98 & 3473.96 & 3472.41 & 3467.87 & 3648.30 & 3648.19 & 3648.87 & 3648.12 \\
\hline$m_{\mathrm{hmax}}$ & 2.0446 & 2.0446 & 2.0446 & 2.0446 & 2.0446 & 2.0694 & 2.0679 & 2.0765 & 2.0792 \\
\hline$m_{\text {shmax }}$ & 2.0072 & 1.9292 & 1.9137 & 1.9125 & 1.9089 & 2.0446 & 2.0446 & 2.0446 & 2.0446 \\
\hline$u_{\mathrm{hmax}}$ & 0.3236 & 0.1068 & 0.1123 & 0.1103 & 0.1050 & 0.1422 & 0.1345 & 0.1241 & 0.1201 \\
\hline$u_{\text {shrmax }}$ & 0.1513 & 0.2832 & 0.1062 & 0.1013 & 0.0883 & 0.1565 & 0.1542 & 0.1486 & 0.1485 \\
\hline
\end{tabular}

connections influence little the design results especially in the case of the shakedown design ( $1^{\text {st }}$ order theory). The moment diagrams of the SHF design are shown in Fig. 4. The dashed lines show the computed required design moments $\left(M_{\mathrm{pl}, \mathrm{Rd}}\right)$.

\section{Conclusion}

It has been shown that by using nonlinear mathematical programming tools, complex optimum design problems can be formulated and solved including elastic-plastic properties and semi rigid connections. It can be seen from the results of Table 3 that within the limits studied $\left(25 E I_{\mathrm{b}} / L_{\mathrm{b}}\right.$ to $\left.8 E I_{\mathrm{b}} / L_{\mathrm{b}}\right)$, the semi rigid connections influence little the response of the structure.

\section{References}

1 Hegedüs L, Iványi M., Use of approximate engineering methods for semirigid frame analysis, Periodica Polytechnica, Civil Engineering 41 (1997), no. 1, 17-38.

2 Lógó J, Kaliszky S, Mohammed Hjiaj, A parametric survey of the influence of the semi-rigid connections on the shakedown of elasto-plastic frames, Periodica Polytechnica, Civil Engineering 52 (2006), no. 2.

3 Gáspár Zs, Nédli P, Global numerical analysis of elastoplastic frames, Computer Assisted Mechanics and Engineering Sciences 5 (1998), 93-100.

4 Design of Steel Structures Part 1-1, General rules for buildings, Brussels, May 2005.

5 Design of joints, Design of Steel Structures Part 1-8, Brussels, May 2005

6 Kaliszky S, Lógó J, Plastic Behaviour and Stability Constraints in the Shakedown Analysis and Optimal Design of Trusses, Journal of Structural and Multidisciplinary Optimization 24 (2002).

7 Wood RH, The Stability of Tall Buildings, Proc. Inst. Civ. Eng., vol. 11, 1958.

8 Nédli P, Elastic-plastic analysis of frames in case geometrical nonlinearity, Acta Technica Acad. Sci. Hung. 107 (1995), no. 1-2, 99-108.

9 Aribert JM, Lachal A, Dinga O, Classification of composite beam-tocolumn connections and consequences for practical design, German Conference on Composite Constructions in Kaiserslautern (1997), pp. 15-26. 